\title{
Inline monitoring of high cell density cultivation of Scenedesmus rubescens in a mesh ultra-thin layer photobioreactor by Photon Density Wave spectroscopy
}

\section{Michael Sandmann ( $\nabla$ sandmann@hs-nb.de)}

University of Applied Sciences Neubrandenburg, Brodaer Straße 2, D-17033 Neubrandenburg, Germany

\section{Marvin Münzberg}

University of Potsdam, Institute of Chemistry, Physical Chemistry - innoFSPEC Potsdam, Am Mühlenberg 3, D-14476 Potsdam, Germany

\section{Lena Bressel}

University of Potsdam, Institute of Chemistry, Physical Chemistry - innoFSPEC Potsdam, Am Mühlenberg 3, D-14476 Potsdam, Germany

\section{Oliver Reich}

University of Potsdam, Institute of Chemistry, Physical Chemistry - innoFSPEC Potsdam, Am Mühlenberg 3, D-14476 Potsdam, Germany

\section{Roland Hass}

University of Potsdam, Institute of Chemistry, Physical Chemistry - innoFSPEC Potsdam, Am Mühlenberg 3, D-14476 Potsdam, Germany; PDW Analytics GmbH, Geiselbergstr. 4, 14476 Potsdam, Germany

\section{Research note}

Keywords: Photon Density Wave spectroscopy, multiple light scattering, algae cultivation, process analytical technology, fiber-optical spectroscopy, MUTL photobioreactor

Posted Date: October 19th, 2021

DOI: https://doi.org/10.21203/rs.3.rs-951382/v1

License: (9) This work is licensed under a Creative Commons Attribution 4.0 International License. Read Full License

Version of Record: A version of this preprint was published at BMC Research Notes on February 15th, 2022. See the published version at https://doi.org/10.1186/s13104-022-05943-2. 


\section{Abstract \\ Objective}

Due to multiple light scattering that occurs inside and between cells, quantitative optical spectroscopy in turbid biological suspensions is still a major challenge. This includes also optical in-line determination of biomass in bioprocessing. Photon Density Wave (PDW) spectroscopy, a technique based on multiple light scattering, enables the independent and absolute determination of optical key parameters of concentrated cell suspensions, which allow to determine biomass during cultivation.

\section{Results}

A unique reactor type, called "mesh ultra-thin layer PBR" was used to create a highly concentrated algal suspension. PDW spectroscopy measurements were carried out continuously in the reactor without any need of sampling or sample preparation, over 3 weeks, and with 10-minutes time resolution. Conventional dry matter content and coulter counter measurements have been employed as established off-line reference analysis. The PBR allowed peak cell dry weight (CDW) of $33.4 \mathrm{~g} \mathrm{~L}^{-1}$. It is shown that the reduced scattering coefficient determined by PDW spectroscopy is strongly correlated with the biomass concentration in suspension and is thus suitable for process understanding. The reactor in combination with the fiber-optical measurement approach will lead to a better process management.

\section{Introduction}

Algae are one of the most promising candidates for solving the problem of nowadays need for renewable energy and sustainable food [1-7]. In comparison to heterotrophic production systems like yeasts, phototrophic production of algae still needs significant improvement to operate cultivations at high cell densities. To close this gap, different types of photo bioreactors (PBR) were devised in the past but upscaling into an industrial scale is still a challenge and the initial investment and operating costs are still high [8]. The optimization of PBR in terms of light harvest, nutrient supply, or gas exchange is of utmost importance for improvement of the biomass production efficiency.

One potential approach to monitor the outcome of PBR design-changes, is the implementation of suitable in-line process analytical technologies (PAT), providing access to algal growth kinetics also at very high cell concentrations. A common approach is to monitor the process state either by dry matter content determination as established off-line analysis or by optical density probes [9-11]. Off-line analyses use aliquots of the cell culture which often increases the risk of contamination with other microbes, is time consuming and thus gives strongly delayed process information. Due to multiple light scattering that occurs inside and between cells, quantitative optical in-line determination of biomass by, e.g., optical density probes, is still a major challenge [11]. 
To evaluate if Photon Density Wave (PDW) spectroscopy [12] would be suitable for process monitoring at high algal cell concentrations, the green alga Scenedesmus rubescens was cultivated in a so-called "mesh ultra-thin layer (MUTL) PBR". The reactor design was recently described [13-15]. However, detailed knowledge about, e.g., multiphase-fluid dynamics, light penetration, or effects of the specialized geometry on algal biology is still missing. Here, this PBR was used to generate a high cell density cultivation with a CDW of $33.4 \mathrm{~g} \mathrm{~L}^{-1}$. PDW spectroscopy was used to investigate biomass dynamics over time. PDW spectroscopy is a fiber-optical measurement technology for the absolute, independent, and calibrationfree determination of the optical coefficients (absorption and reduced scattering coefficient, $\mu_{\mathrm{a}}$ and $\mu_{\mathrm{s}}{ }^{\prime}$, respectively) of liquid dispersions. It has been recently applied to numerous chemical, physical, and biotechnological processes [16-22].

\section{Experimental Section}

\section{Photo bioreactor}

Algal cultivation was performed under greenhouse conditions in a prototype MUTL PBR at the IGV GmbH (Nuthetal, Germany) during August and September. A scheme of the PBR is displayed in figure $1 \mathrm{~A}$ and the adapter for the PDW spectroscopy process probe is shown in figure $1 \mathrm{~B}$.

Briefly, the configuration of the PBR used in this work consists of 40 vertically stacked horizontal polymer nets. The polymer nets are enclosed in a $2.5 \mathrm{~m}$ high, transparent polymethyl methacrylate housing, occupying a footprint area of $4.84 \mathrm{~m}^{2}$. The water-cooled double bottom of the reactor is coupled to an external cooling device. The PBR is equipped with a recirculation loop to pump algal suspension from the sink to the top of the PBR from which the suspension is sprayed on the polymer nets. During their movement through the stack of nets, cells are exposed to the incident light and the surrounding gas phase. The PDW spectroscopy probe is inserted in a special flow-cell implemented in the recirculation loop (Fig. 1 B). Nitrate content was measured during algal growth with the 'Nitrate Cell Test-Kit in seawater' from WTW (Xylem Analytics Germany Sales GmbH \& Co. KG, WTW, Weilheim, Germany) and a concentrated solution of all five macronutrients were added if around $40 \%$ of the starting concentration of nitrate was reached during the experiment. This procedure was based on the assumption that all macro constituents were depleted in parallel. During the cultivation, biomass growth was supported through additional 16 high pressure sodium lamps with 400 watts power each.

\section{Culturing of Scenedesmus rubescence}

The preculturing was done in ten lab-scale bubble columns ( $1.8 \mathrm{~L}$ suspension volume each) under continuous light with a light intensity of $120 \mu \mathrm{mol}$ photons $\mathrm{m}^{-2} \mathrm{~s}^{-1}$ at $25^{\circ} \mathrm{C}$ and $3 \% \mathrm{CO}_{2}[\mathrm{v} / \mathrm{v}]$ in a synthetic growth media ( $1 \frac{1}{2}$ Tamiya [23]). Further up-scaling for production of the inoculum culture for the 
cultivation experiment within the MUTL PBR was done under greenhouse conditions in a $90 \mathrm{~L}$ tubular PBR with the same growth media.

\section{Quantification cell size and cell concentration}

Cell size and cell concentration were determined by offline measurements (MULTISIZER 3, Beckman Coulter, Krefeld, Germany) once or twice per day. An important parameter that can be analyzed by this kind of device is the total cellular volume (given in cubic micrometers $\left(\mu \mathrm{m}^{3}\right)$ per milliliter of suspension). It is defined as the sum of all cellular volumes present in $1 \mathrm{~mL}$ of suspension and is a measure of the biomass or wet-weight concentration of the cells present in suspension.

\section{Determination of the cell dry weight}

Determination of CDW was done gravimetrically once or twice per day. Briefly, 5 to $10 \mathrm{~mL}$ aliquots from the cell suspension were centrifuged at 3,000 $\mathrm{g}$ f for $10 \mathrm{~min}$ in pre-weighed glass tubes. The pellet was afterwards washed with deionized water and then dried at $105^{\circ} \mathrm{C}$ for $24 \mathrm{~h}$. Prior to the weight measurements, the hot glass tubes were cooled to room temperature in a desiccator. The weight difference corresponds to the dry matter content of the cell suspension.

\section{Photon Density Wave spectroscopy}

A PDW spectrometer features an intensity modulated laser as light source. By an optical emission fiber, the laser light is guided into a fiber-optical probe being implemented in a special flow cell [25] in the recirculation loop. The fiber end acts as point-like light source. Due to multiple light scattering and absorption a PDW is created within the algae suspension. Further detection fibers in the probe collect light from the PDW and guide it back to the detector inside the spectrometer. Changes to phase and amplitude of the PDW are characterized by a vector network analyser within the spectrometer. These changes give access to the optical coefficients of the algae suspension.

Here, a self-constructed PDW spectrometer (commercial versions available by PDW Analytics GmbH, Potsdam, Germany) was applied [25] and a measurement wavelength of $906 \mathrm{~nm}$ with modulation frequencies from $10 \mathrm{MHz}$ to $810 \mathrm{MHz}$ was used. To characterize the PDW as function of distance between emission and detection fiber, a fiber-optical probe consisting of one central emission fiber and 12 surrounding detection fibers was constructed [25]. The distances ranged from $9.47 \mathrm{~mm}$ to $20.38 \mathrm{~mm}$. For raw data analysis, a refractive index of $n=1.3264$ at $906 \mathrm{~nm}$ was used. 


\section{Results}

Development of the algal culture in the MULT PBR is shown in Fig. 2A. Algal cells showed a lack phase of 2 to 3 days and afterwards started strong biomass growth with nearly linear increase of dry matter content in the cell suspension. Close to the end of the lack phase the artificial light was switched on (Fig. 2A). Mean growth rate was comparatively high $\left(2.39 \mathrm{~g} \mathrm{~L}^{-1}\right.$ day $\left.{ }^{-1}\right)$ during first 14 days of growth. At day 14 the cell suspension was partly harvested and refilled with fresh culture media (Fig. 2A) and afterwards the reactor was running for additional 6 days. At different timepoints, concentrated solution of macro nutrients was added to the cell culture (Fig. 2A). The analysis of total cellular volume has been used as additional reference technique. It exhibits a very similar development like the dry matter content. PDW spectroscopy gathered the optical coefficients with high temporal resolution, unattended, and without sampling. Generally, $\mu_{\mathrm{s}}^{\prime}$ at $906 \mathrm{~nm}$ was able to follow the development of both reference analyses (Fig. 2A). Based on $\mu_{\mathrm{s}}$ ', dilution effects from the addition of concentrated media component solution and the harvesting procedure can be visualised with high time resolution and even growth rates are obtained in nearly real-time (Additional file 1: Figure S1). However, at low CDW PDW spectroscopy is not able to provide reliable optical coefficients due to too low turbidity of the algae suspension. Based on Coulter counter measurements, a deeper analysis of the population dynamics of the algal cells can be obtained (Fig. 2B). During cultivation, cells increased their mean diameter from approximately 4.0 to $5.6 \mu \mathrm{m}$ (Fig. 2B). Also, the coefficient of variation (C.V.Size of the cell size is displayed. The C.V.Size is a relative measure for the widths of a distribution and can be used to quantify the cell-to-cell heterogeneity inside a cell population. The C.V. .size increased from 0.27 to 0.37 , which means an increased heterogeneity. This is visible also in the cell size distributions (Fig. 2C). Cell size ranged between 2 to $12 \mu \mathrm{m}$ in diameter, which is similar to other Scenedesmus species [24].

$\mu_{\mathrm{s}}$ 'values have been correlated with both corresponding reference analyses, indicating a linear correlation with $\mathrm{R}^{2}=0.9726$ against $\mathrm{CDW}$ and $\mathrm{R}^{2}=0.9561$ against Coulter counter data (Fig. 3). Correlation of both reference analyses resulted in $\mathrm{R}^{2}=0.9895$ (Additional file 1: Figure S2).

\section{Discussion}

Quantitative optical in-line spectroscopy is still a challenge especially in concentrated biotechnical process [26]. One reason is a parallel occurrence of light absorption and multiple light scattering. Thus, optical methods are required which can separate light absorption and scattering also for very turbid materials.

PDW spectroscopy is such an advanced process analytical technology which allows for the independent and absolute determination of optical key parameters of concentrated cell suspensions $[11,26]$. As model processes, photoautotrophic growth of algal cells has been investigated in a very specialized photobioreactor called MUTL (Fig. 1). In comparison to classical PBRs, MUTL PBRs exhibit significantly higher peak CDW and growth rates. This was shown in three different references, but with a strong spread of the exactly reached peak CDW ranging from 6.6 to $40 \mathrm{~g} \mathrm{~L}^{-1}$ [13-15]. In this study, the MUTL PBR was 
used to culture high cell densities up to $33.4 \mathrm{~g} \mathrm{~L}^{-1}$. A configuration of the MUTL PBR was used with 40 nets inside the net-matrix and the biomass growth was supported through 16 additional high pressure sodium lamps with 400 watts power each. Conventional off-line techniques have been used to describe growth characteristics of the culture. For comparison, in-line measurements by PDW spectroscopy were performed directly in the cell culture. PDW spectroscopy provided reduced scattering coefficients of the algal suspension. In this experiment, $\mu_{\mathrm{s}}$ 'is found to be strongly correlating with both applied reference analyses (Fig. 2 and Fig. 3). PDW spectroscopy was able to measure fast dynamics, e.g., harvesting of the cells and influences of nutrient addition. In comparison to off-line analyses, PDW spectroscopy was able to continuously monitor fast changes in the cell suspension, without any need for sampling or sample preparation and with high temporal resolution. It has been shown that also growth rates derived from $\mu_{\mathrm{s}}^{\prime}$ can be calculated (Additional file 1: Figure S1).

PDW spectroscopy is able to monitor dynamics in processes in much more concentrated suspensions or dispersions. Examples are, e.g., concentrated nanoparticle suspensions with $\mu_{\mathrm{s}}$ ' of up to $20 \mathrm{~mm}^{-1}$ or emulsification processes with $\mu_{\mathrm{s}}$ ' of more than $5 \mathrm{~mm}^{-1}$ [16]. In a biotechnological production of polyhydroxyalkanoate (PHA), CDW of $40 \mathrm{~g} \mathrm{~L}^{-1}$ have been reached corresponding to $\mu_{\mathrm{s}}^{\prime}$ of $2 \mathrm{~mm}^{-1}$ [22]. Thus, for monitoring algae growth, the current upper limitation is the achievable biomass concentration, not the applicability of the PDW technology.

The determined optical parameters of algal cells can be used in future approaches to model light penetration within PBRs which is expected to be a key to enhance reactor performance $[27,28]$. Thus, there is a high potential for PDW spectroscopy to be used for advanced process monitoring, process control, and to ensure a better process understanding in concentrated biotechnological suspensions.

\section{Limitations}

- Data from reference analysis were taken without replicates.

- Cultivation reproducibility with respect to CDW of $33.4 \mathrm{~g} \mathrm{~L}^{-1}$ was not tested. However, focus here was on suitability of PDW spectroscopy for in-line biomass monitoring.

- Raw data analysis for PDW spectroscopy took assumptions into account, referring to the refractive index and volume fraction of cells, respectively.

- The flow cell for PDW spectroscopy probe implementation was not optimized for reduction of shear forces onto cells. Thus, structural damage might have been induced.

\section{Abbreviations}

PDW

Photon Density Wave

CDW

cell dry weight 
photobioreactor

$\min$

minute

C.V.

coefficient of variation

$\mathrm{GR}_{\mathrm{PDW} \text {,in-line }}$

Growth rate based on $\mu s^{\prime}$

\section{References}

1. de Jaeger L, Verbeek RE, Draaisma RB, Martens DE, Springer J, Eggink G, Wijffels RH. Superior triacylglycerol (TAG) accumulation in starchless mutants of Scenedesmus obliquus: (I) mutant generation and characterization. Biotechnol Biofuels. 2014;7:69.

2. Sandmann M, Schafberg M, Lippold M, Rohn S. Analysis of population structures of the microalga Acutodesmus obliquus during lipid production using multi-dimensional single-cell analysis. Sci Rep. 2018;8:6242.

3. Wells ML, Potin P, Craigie JS, Raven JA, Merchant SS, Helliwell KE, Smith AG, Camire ME, Brawley SH. Algae as nutritional and functional food sources: revisiting our understanding. J Appl Phycol. 2017;29:949-82.

4. Zhu L. Biorefinery as a promising approach to promote microalgae industry: An innovative framework. Renew Sust Energ Rev. 2015;41:1376-84.

5. Smetana S, Sandmann M, Rohn S, Pleissner D, Heinz V. Autotrophic and heterotrophic microalgae and cyanobacteria cultivation for food and feed: Life Cycle Assessment. Bioresour Technol. 2017;245:162-70.

6. Sevgili H, Sezen S, Yılayaz A, Aktaş Ö, Pak F, Aasen IM, Reitan KI, Sandmann M, Rohn S, Turan G, Kanyılmaz M. Apparent nutrient and fatty acid digestibilities of microbial raw materials for rainbow trout (Oncorhynchus mykiss) with comparison to conventional ingredients. Algal Res. 2019;42:101592.

7. Hensel B, Jakop U, Scheinpflug K, Schröter F, Sandmann M, Mühldorfer K, Schulze M. Low temperature preservation: influence of putative bioactive microalgae and hop extracts on sperm quality and bacterial load in porcine semen. Sustain Chem Pharm. 2021;19:100359.

8. Gupta PL, Lee SM, Choi HJ. A mini review: photobioreactors for large scale algal cultivation. World J Microbiol Biotechnol. 2015;31:1409-17.

9. Sonnleitner B, Locher G, Fiechter A. Biomass determination. J Biotechnol. 1992;25:5-22.

10. Gregory ME, Thornhill NF. The effects of aeration and agitation on the measurement of yeast biomass using a laser turbidity probe. Bioprocess Eng. 1997;16:339-44. 
11. Münzberg M, Hass R, Dinh Duc Khanh N, Reich O. Limitations of turbidity process probes and formazine as their calibration standard. Anal Bioanal Chem. 2017;409:719-28.

12. Bressel L, Hass R, Reich O. Particle sizing in highly turbid dispersions by Photon Density Wave spectroscopy. J Quant Spectrosc Radiat Transf. 2013;126:122-9.

13. Pulz O, Broneske J, Waldeck P. IGV GmbH Experience Report, Industrial Production of Microalgae Under Controlled Conditions: Innovative Prospects. In: Handbook of Microalgal Culture: Applied Phycology and Biotechnology. West Sussex: John Wiley \& Sons;: Chichester; 2013. pp. 445-60.

14. Schreiber C, Behrendt D, Huber G, Pfaff C, Widzgowski J, Ackermann B, Müller A, Zachleder V, Moudř́ková Š, Mojzeš P, Schurr U, Grobbelaar J, Nedbal L. Growth of algal biomass in laboratory and in large-scale algal photobioreactors in the temperate climate of western Germany. Bioresour Technol. 2017;234:140-9.

15. Sandmann M, Smetana S, Heinz V, Rohn S. Comparative life cycle assessment of a mesh ultra-thin layer photobioreactor and a tubular glass photobioreactor for the production of bioactive algae extracts. Bioresour Technol. 2021;340:125657.

16. Hass R, Münzberg M, Bressel L, Reich O. Industrial applications of Photon Density Wave spectroscopy for in-line particle sizing [Invited]. Appl Opt. 2013;52:1423-31.

17. Vargas Ruiz S, Hass R, Reich O. Optical monitoring of milk fat phase transition within homogenized fresh milk by Photon Density Wave spectroscopy. Int Dairy J. 2012;26:120-6.

18. Werner P, Münzberg M, Hass R, Reich O. Process analytical approaches for the coil-to-globule transition of poly(N-isopropylacrylamide) in a concentrated aqueous suspension. Anal Bioanal Chem. 2017:409:807-19.

19. Hartwig A, Hass R. Monitoring Lactose Crystallization at Industrially Relevant Concentrations by Photon Density Wave Spectroscopy. Chem Eng Technol. 2018;41:1139-46.

20. Häne J, Brühwiler D, Ecker A, Hass R. Real-time inline monitoring of zeolite synthesis by Photon Density Wave Spectroscopy. Microporous Mesoporous Mater. 2019;288:109580.

21. Bressel K, Müller W, Leser ME, Reich O, Hass R, Wooster TJ. Depletion-Induced Flocculation of Concentrated Emulsions Probed by Photon Density Wave Spectroscopy. Langmuir. 2020;36:350413.

22. Gutschmann B, Schiewe T, Weiske MTH, Neubauer P, Hass R, Riedel SL. In-Line Monitoring of Polyhydroxyalkanoate (PHA) Production during High-Cell-Density Plant Oil Cultivations Using Photon Density Wave Spectroscopy. Bioengineering. 2019;6:85.

23. Hase $E$, Morimura $Y$, Tamiya $H$. Some data on the growth physiology of Chlorella studied by the technique of synchronous culture. Arch Biochem Biophys. 1957;69:149-65.

24. Sandmann M, Lippold M, Saalfrank F, Odika CP, Rohn S. Multidimensional single-cell analysis based on fluorescence microscopy and automated image analysis. Anal Bioanal Chem. 2017;409:4009-19.

25. Hass R, Reich O. Photon Density Wave Spectroscopy for Dilution-Free Sizing of Highly Concentrated Nanoparticles During Starved-Feed Polymerization. Chem Phys Chem. 2011;12:2572-5. 
26. Hass R, Munzke D, Ruiz SV, Tippmann J, Reich O. Optical monitoring of chemical processes in turbid biogenic liquid dispersions by Photon Density Wave spectroscopy. Anal Bioanal Chem. 2015;407:2791-802.

27. Posten C. Design principles of photo-bioreactors for cultivation of microalgae. Eng Life Sci. 2009;9:165-77.

28. Luzi G, McHardy C, Lindenberger C, Rauh C, Delgado. Comparison between different strategies for the realization of flashing-light effects - Pneumatic mixing and flashing illumination. Algal Res. 2019;38:101404.

\section{Declarations}

\section{Ethics approval and consent to participate}

Not applicable.

\section{Consent for publication}

Not applicable.

\section{Availability of data and material}

The original datasets and other miscellaneous materials related to the investigation are available upon reasonable request to the corresponding author.

\section{Competing interests}

The authors declare that they have no conflict of interests.

\section{Funding}

We like to thank the "Centre for Innovation Competence" innoFSPEC of the BMBF (grant 03Z2AN12) and the Land Brandenburg for the project SEMIRAMIS (ILB project number 80139585 ) for financial support. 


\section{Author's contributions}

MS, MM, LB, and RH conducted the experiment, collected data, and analyzed the dataset. OR contributed with supervision and gathering of the funds. MS and $\mathrm{RH}$ prepared the manuscript. All authors read and approved the final manuscript.

\section{Acknowledgements}

We like to acknowledge IGV GmbH, especially Thomas Wencker and Peter Waldeck, for the possibility to use their photobioreactors for our measurements as well as Till Meiling and Carsten Hille for experimental help.

\section{Figures}

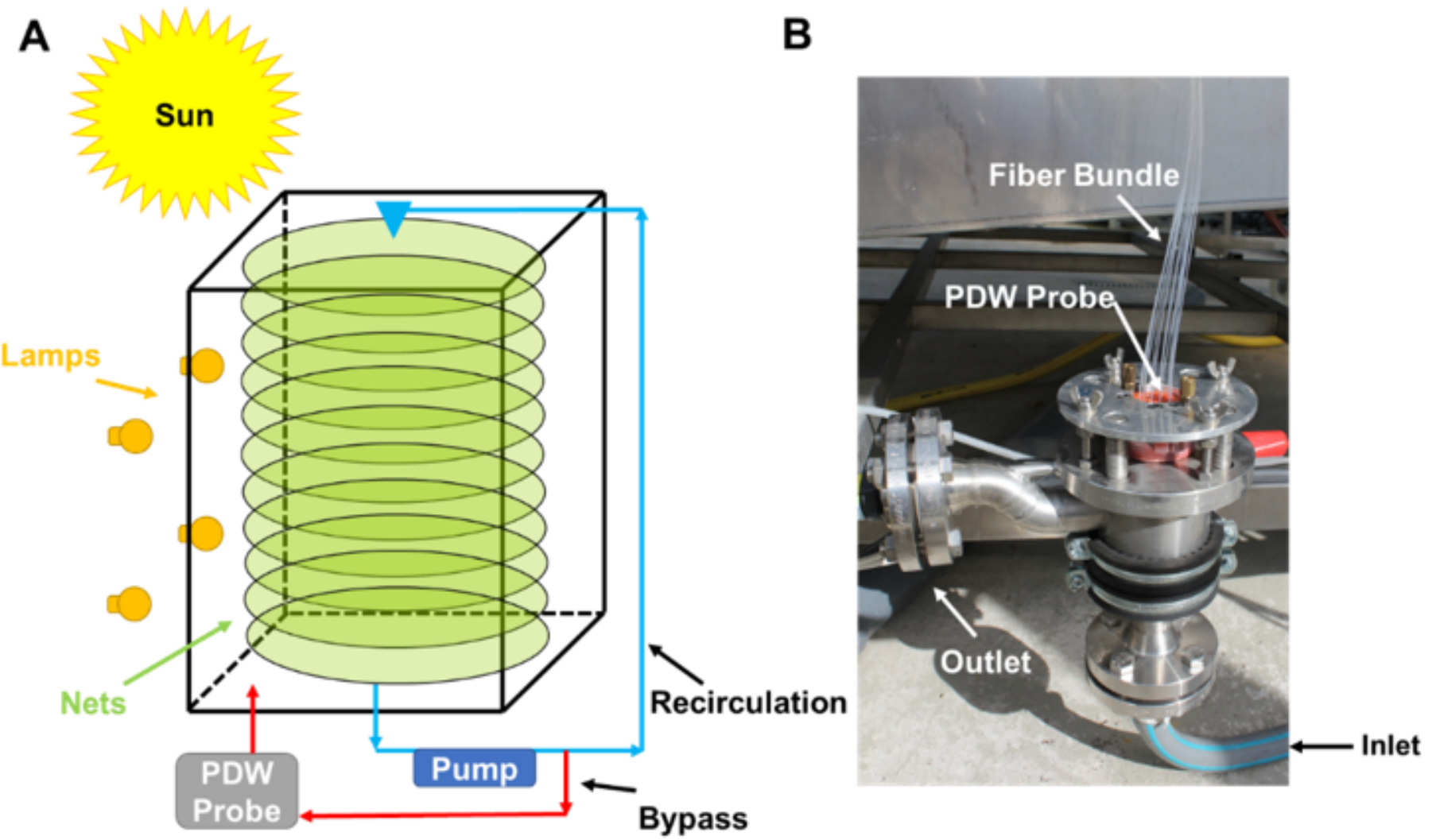

Figure 1 
Experimental setup. A Schematic drawing of the MUTL PBR including a PDW spectroscopy probe. B Photograph of the PDW probe mounted on the MUTL PBR recirculation loop.

A

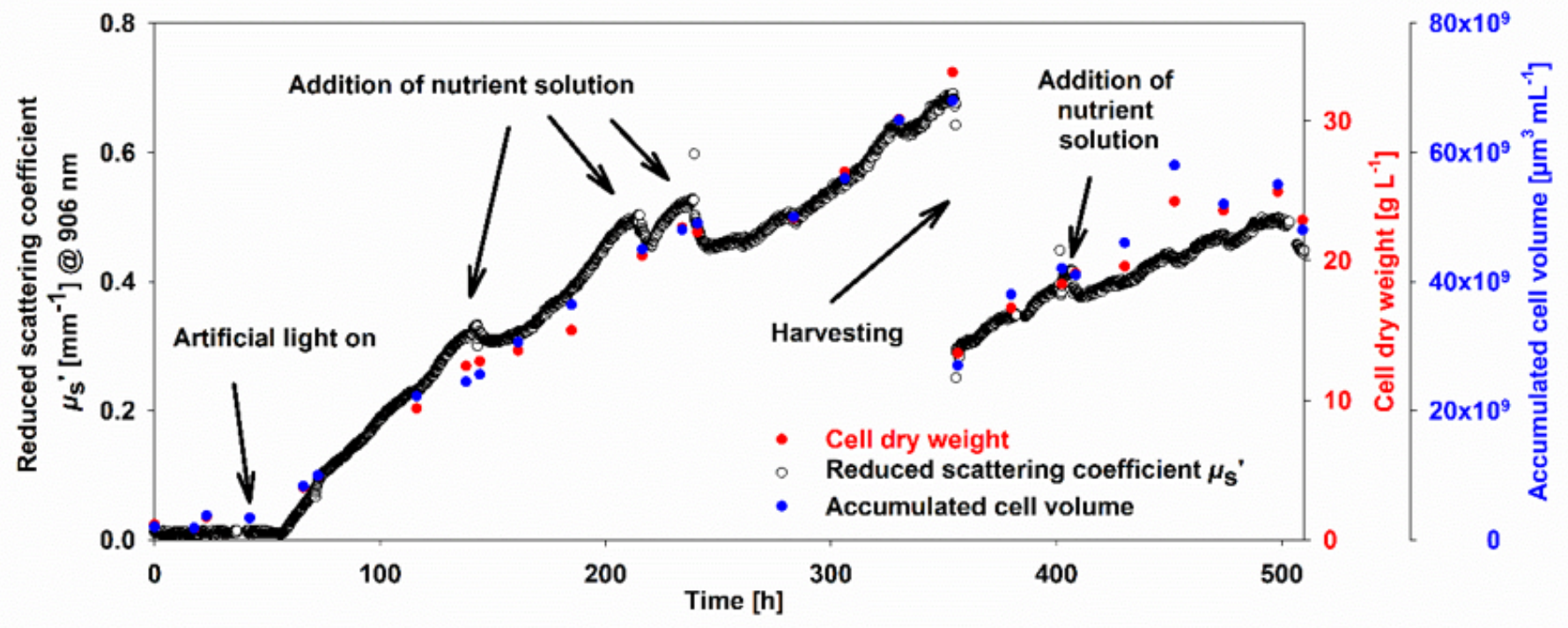

B
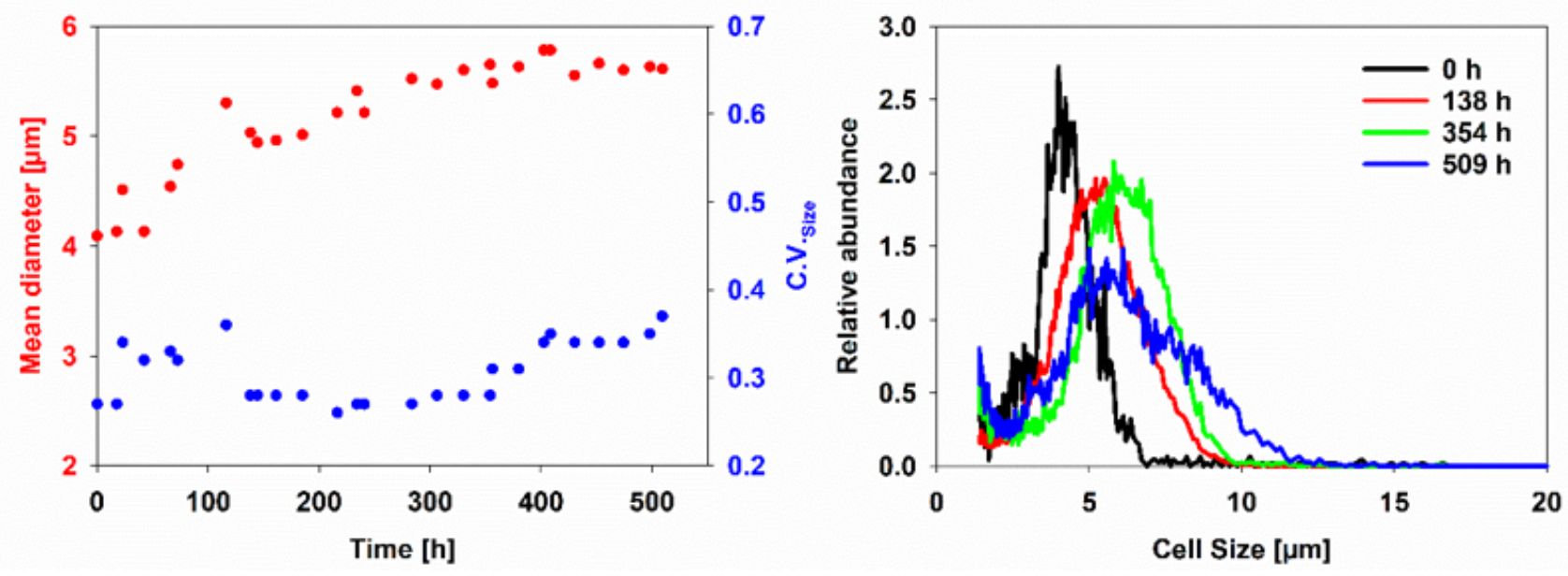

Figure 2

Culture characterization over time. A Development of biomass related parameters. B Mean diameter and coefficient of variation (C.V.) as a measure for the width of the cell size distribution. C Selected cell size distributions determined by Coulter counter (sample time in legend). 


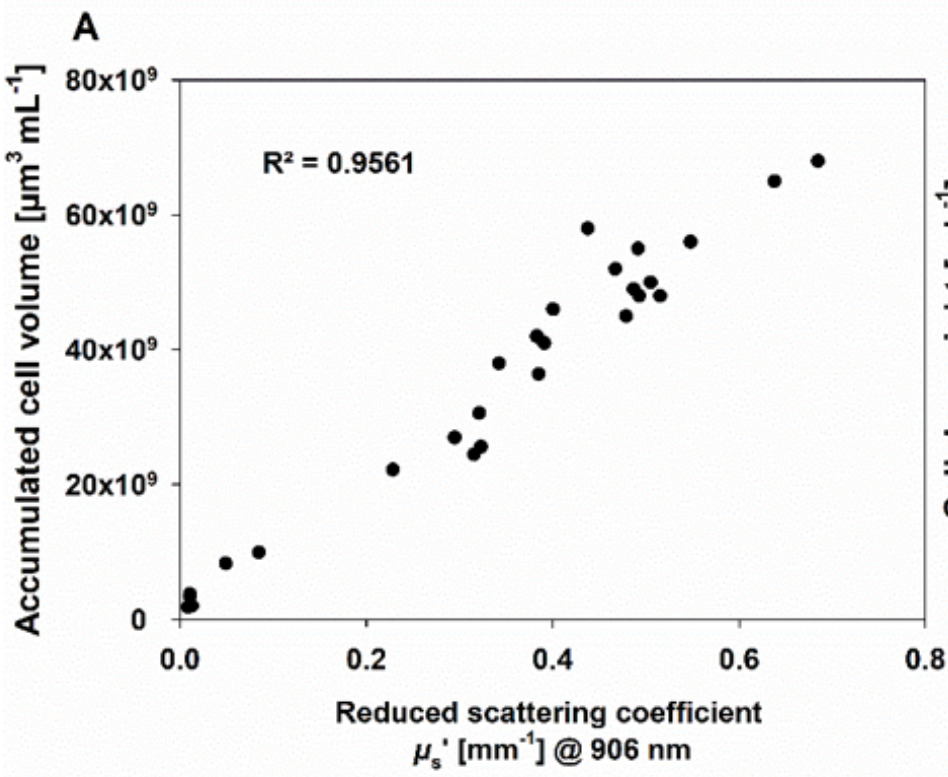

\section{B}

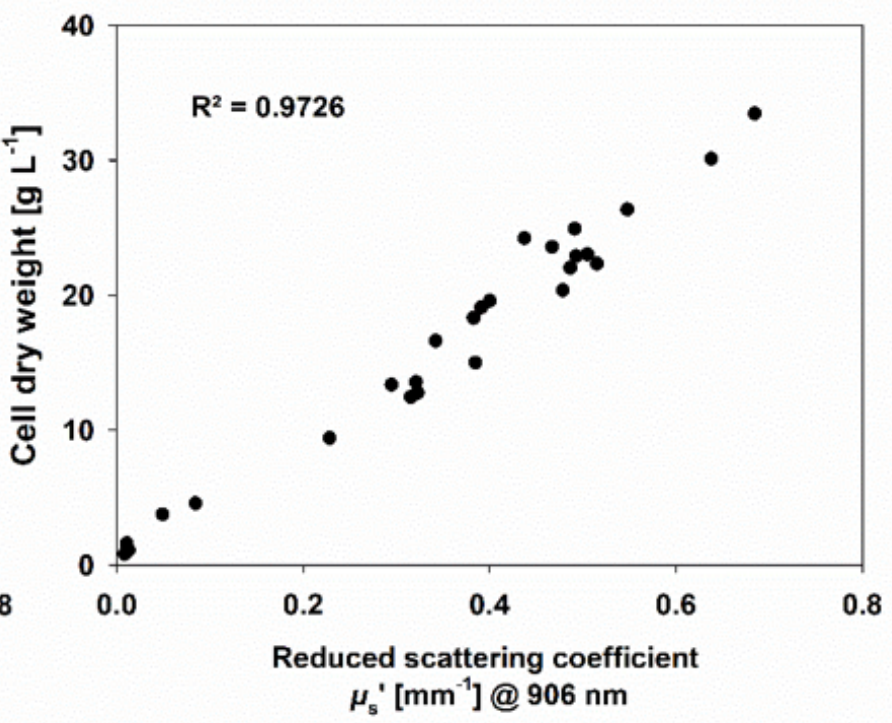

Figure 3

Correlation of PDW spectroscopy with reference analyses. A Correlation of $\mu s^{\prime}$ at $906 \mathrm{~nm}$ from PDW spectroscopy with accumulated cellular volume derived from Coulter counter measurements. B Correlation of $\mu s^{\prime}$ at $906 \mathrm{~nm}$ from PDW spectroscopy with CDW.

\section{Supplementary Files}

This is a list of supplementary files associated with this preprint. Click to download.

- SupplementalMaterial.docx 